\title{
26th Texas Symposium on Relativistic Astrophysics
}

Published online: 16 November 2013

(C) Sociedade Brasileira de Física 2013

The 26th Texas Symposium on Relativistic Astrophysics (Texas 2012) was held in São Paulo, Brazil, on 15-20 December 2012. This was the second time this traditional and important symposium had taken place in the southern hemisphere and the first time in South America. It was an honor for us in Brazil to host such a symposium. We thank very much the International Organizing Committee of Texas Symposia for such an opportunity.

As in previous editions, Texas 2012 covered the recent developments in Cosmology, Gravitation, Astroparticle Physics, and related areas of Relativistic Astrophysics. In the website of Texas 2012, http://www.das.inpe.br/texas2012sp, one can find additional details on the presented topics.

It is worth stressing that Texas 2012 had 26 plenary speakers, who reviewed different topics related to relativistic astrophysics. Almost all the plenary presentations can be found at the webpage http://www.das.inpe.br/texas2012sp/ program.php. We take this opportunity to thank all Texas 2012 speakers.

In this issue of the Brazilian Journal of Physics, one finds a collection of selected papers written by plenary speakers. We thank Dr. Harry Blom for making possible the publication of this special issue.

A number of parallel sessions were also held, which covered Cosmology, Galactic and Intergalactic Astrophysics, Compact Objects, High Energy Astrophysics, Astroparticle Physics, Alternative Models and Theories, New Windows, Gravitational Waves, New Projects and Missions, Quantum Effects in Relativistic Astrophysics, and Instrumentation for
Relativistic Astrophysics. We thank very much the chairs of the parallel sessions for their help in drawing the scientific program.

Texas 2012 also had three talks targeting the general public. We thank very much George Matsas, Martin Makler, and Jorge Horvath for accepting to talk in these public sessions.

It would be impossible for Brazil to host Texas 2012 without the financial support given by the Brazilian agencies CAPES, FAPESP, and CNPq. We thank them very much for the support. We also thank IUPAP for its financial support.

On behalf of the Local Organizing Committee, we thank very much the excellent work done by the Scientific Organizing Committee (see http://www.das.inpe.br/texas2012sp/ organizingcommittee.php) chaired and co-chaired by Drs. Odylio Aguiar and Jorge Horvath, respectively.

The organization of a symposium is only possible with the active participation of the Local Organizing Committee (see http://www.das.inpe.br/texas2012sp/organizingcommittee. php). We thank all my colleagues for their spirit of collaboration. Among them, César Costa must be mentioned for his extremely valuable help on many issues.

Last but not least, we thank the Brazilian Physical Society, the Brazilian Astronomical Society, the São Paulo Convention \& Visitors Bureau, and our secretaries (headed by Valéria Fernandes) for their invaluable support.

Jose Carlos N. de Araujo (DAS/INPE/MCTI)

Chair of the Local Organizing Committee

Texas 2012-São Paulo, SP, Brazil 